\title{
Presentation format effects in working memory: The role of attention
}

\author{
PAUL W. FOOS and PAULA GOOLKASIAN \\ University of North Carolina, Charlotte, North Carolina
}

\begin{abstract}
Four experiments are reported in which participants attempted to remember three or six concrete nouns, presented as pictures, spoken words, or printed words, while also verifying the accuracy of sentences. Hypotheses meant to explain the higher recall of pictures and spoken words over printed words were tested. Increasing the difficulty and changing the type of processing task from arithmetic to a visual/ spatial reasoning task did not influence recall. An examination of long-term modality effects showed that those effects were not sufficient to explain the superior performance with spoken words and pictures. Only when we manipulated the allocation of attention to the items in the storage task by requiring the participants to articulate the items and by presenting the stimulus items under a degraded condition were we able to reduce or remove the effect of presentation format. The findings suggest that the better recall of pictures and spoken words over printed words result from the fact that under normal presentation conditions, printed words receive less processing attention than pictures and spoken words do.
\end{abstract}

In the present work, we address the question of why some types of presentation formats result in better recall from working memory than other types do (Goolkasian $\&$ Foos, 2002). We studied the advantages of pictures and spoken words over printed words by varying the type and difficulty of the processing task and manipulating the allocation of attention to presented items in the storage task. We propose that the advantages of pictures and spoken words are largely a result of the allocation of attentional resources.

Some of the early work in which the effects of different formats in the visual modality were compared concentrated on picture-word differences (Goolkasian \& Park, 1980; Kroll \& Corrigan, 1981; Pellegrino, Rosinski, Chiesi, \& Siegel, 1977; Smith \& Magee, 1980) and showed better memory for pictures than for words. Paivio's $(1971,1975,1978)$ dual-coding theory influenced this work and much of our later thinking with respect to picture-word differences. Items can be coded as words and as images. Of course, the differences obtained in these early studies were largely the result of encoding processes and retrieval from long-term memory, whereas in the present work our focus is on working memory.

Other early work was conducted to examine auditoryvisual modality differences (Greene, 1985; Greene, Elliott,

Some of these findings were reported at the 44th Annual Meeting of the Psychonomic Society in Vancouver, November 2003. Our work was supported in part by funds from the foundation of the University of North Carolina at Charlotte and from the State of North Carolina. We thank Bradford Bugni, Melissa Schoolcraft, and Kathy Littman for their assistance with data collection and data analysis. We also extend our thanks to Robert Greene, John Gardiner, Catherine Penney, and an anonymous reviewer for their helpful comments on an earlier version of this article. Correspondence concerning this article should be addressed to P. W. Foos, Department of Psychology, University of North Carolina, 9201 University City Blvd., Charlotte, NC 28223 (e-mail: pwfoos@email.uncc.edu).
\& Smith, 1988; Penney, 1989) and showed that auditory presentation is superior to visual presentation, particularly for the last several items in a list of to-be-remembered items. This finding is known as the modality effect and consists of a sustained superiority for items presented in the auditory mode (i.e., the long-term modality effect) and a brief advantage, due to echoic storage, that can be eliminated by presentation of a similar auditory item immediately following the list (i.e., the suffix effect; Crowder, 1972). The long-term modality effect takes place in long-term memory and is found for both serial and free recall (Cowan, Saults, Elliott, \& Moreno, 2002; Gardiner, Gardiner, \& Gregg, 1983; Greene \& Crowder, 1986). In this important work, however, the effects of pictures, relative to spoken and printed words, was not examined.

In our recent work (Goolkasian \& Foos, 2002), we examined working, rather than long-term, memory for material presented in these different modalities (auditory vs. visual) and in different formats in the same modality (picture vs. printed word) with a dual task that measured the cognitive demands of storage and processing. Participants attempted to remember concrete nouns, presented as printed words, spoken words, or pictures, while verifying the accuracy of math sentences. The results showed that items presented as spoken words or as pictures produced better recall and recognition than printed words did. Reaction time (RT) and accuracy on the processing component of the dual task were maintained at a relatively stable rate despite variation in modality/format for the to-be-remembered items. These results suggest that the previously found advantages of pictures over printed words and of spoken words (auditory presentation) over printed words may be due to the same mechanism(s).

In the present study, we explore explanations for these effects of presentation format. We will refer to the ad- 
vantage of spoken words and pictures over printed words as an effect of presentation format, even though a change in modality is a part of this effect. One possibility for the findings obtained is that the processing task affects item recall. For example, the processing task may draw some resources from the storage of the printed words. While participants are processing the math facts, some intermediate results may be stored verbally. If this were the case, the words stored in the verbal buffer would be susceptible to interference from the processing task, whereas the pictures would be stored in the visual spatial sketchpad without such interference (Baddeley \& Hitch, 1974; Baddeley \& Logie, 1999). Spoken words may not be as disadvantaged by the interference from the processing task, because of the long-term modality effect. Varying the difficulty, as well as the kind of processing task, would help to determine how important the processing task is in producing the underlying effect of presentation format. In Experiment 1, we tested whether increases in the difficulty of the processing task would result in an even greater difference between printed words and the other modality/format conditions, and in Experiment 2, we used a reasoning task that was based on visual judgments, rather than the math task used in our previous work. If the effects of presentation format remained relatively unaffected by these changes, it would eliminate the processing task as a major reason for the presentation format effects that have been consistently obtained in our previous work.

In Experiment 2, we also examined whether the effect of presentation format may, in part, be due to the longterm modality effect. The data were reanalyzed in order to compare recall of the last item presented on each trial of the storage task across the three presentation format conditions. If the modality effect underlies the effect of presentation format, we would expect to find that recall of the last item varies substantially across format conditions, with the spoken words being recalled at a higher rate than the printed words. Moreover, if the effect is a genuine modality effect, we would also expect to find spoken words recalled at a higher rate than pictures.

Finally, one might hypothesize that the differences obtained are a result of the differential allocation of attentional resources to pictures, spoken words, and printed words. Pictures and spoken words may receive more processing. Early work by Posner, Nissen, and Klein (1976) provided some evidence for differential attention effects by modality. They found that visual signals are less alerting than stimuli in other modalities and suggested that auditory signals had an advantage because of an automatic alerting effect. Others have shown similar differences in selective attention to visual and auditory stimuli (Melara \& O’Brien, 1987; Patching \& Quinlan, 2002). More recent work, however, has shown that the alerting effect is dependent on the central allocation of attention and that visual, as well as auditory, stimuli can produce the same effect (Turatto, Benso, Galfano, \& Umiltà, 2002).

Findings such as these suggest that a reason for the lower performance with printed words than with pictures and spoken words may be that printed words receive less effortful processing (Hasher \& Zacks, 1979). Spoken words and pictures call for attentional resources and effortful processing, whereas printed words receive less effortful processing and are, thus, remembered less well. This hypothesis was tested in Experiment 3 by requiring that the participants articulate the items presented in the storage task. The very fact of articulating the items should force the participants to pay attention to all of the items, and as a result, the effect of presentation format should be diminished. In Experiment 4, the attention hypothesis was tested a second time by comparing recall of degraded items with recall of normal items. Degrading the items would make them more difficult to discern and would force the participants to pay more attention to them in order to remember them. Again, our prediction was that the effect of presentation format would be diminished with the degraded items, in comparison with the normal items.

The dual task used in these experiments is modeled on a complex operations span task (La Pointe \& Engle, 1990) and was used in our previous work (Goolkasian \& Foos, 2002, 2005). Our processing component was a sentence verification task, and it included articulation of the stimulus sentences. Articulation has been shown (Goolkasian $\&$ Foos, 2002) to eliminate the sound advantage due to echoic memory. Performance on the processing task was recorded by measuring RT and accuracy to each sentence. The storage component required the participants to store either three or six items, presented as pictures, spoken words, or printed words, and to free recall them at the end of a trial.

\section{EXPERIMENT 1}

In this first experiment, we varied the difficulty of the processing task to determine whether format effects would be influenced by the resources devoted to processing. Some early theories of working memory assumed that all the operations taking place in working memory used one common pool of resources (the central executive) that was limited in capacity (Baddeley \& Hitch, 1974; Daneman \& Carpenter, 1980). If this was the case, it is possible that processing items that varied in modality/format required different amounts of central executive capacity, and as a result, the effects of presentation format may have been a by-product of the processing-storage tradeoff. Increasing the resources devoted to processing, by making the processing task more difficult, should decrease performance on the storage task. The effects of presentation format should, then, be even more pronounced than in our original findings.

If, on the other hand, the effect of presentation format remains relatively unaffected by the difficulty of the processing task, we can eliminate this variable as an important influence on format differences. Some recent theories of working memory have suggested that separate resources are used in the storage and processing components of working memory and that these tasks can be in- 
dependent (Oberauer, 2002; Oberauer, Demmrich, Mayr, \& Kliegl, 2001), or that resource sharing can be time dependent, rather than based on the difficulty of the processing task (Barrouillet, Bernardin, \& Camos, 2004).

\section{Method}

Participants. The participants were 26 men and women students drawn from the University of North Carolina at Charlotte. They were volunteers who participated in the experiment to obtain extra credit points toward their psychology class grade.

Materials. The 54 items used are listed in the Appendix. Each of these appeared as a printed word, a spoken word, and a picture. The items used as stimuli were chosen from a pool of several hundred clip art photos on the basis of ratings by an independent group of 40 students drawn from the same population as the participants. An item was chosen for the study if at least $90 \%$ of the raters agreed on a name for the picture. Each clip art picture was imported into Adobe Photoshop, and its size was adjusted to approximately $4 \times 4 \mathrm{~cm}$ for use in all the experiments reported. When nouns appeared as printed words, they were uppercase characters printed with a Geneva font in a character size of 24-point type. Spoken words were sound files created by a human female voice as a Macintosh system sound file.

Sentences used for the processing task were questions adapted from La Pointe and Engle (1990). Processing difficulty was manipulated by varying the number of mathematical operations present in these sentences. Easy sentences contained two components: the multiplication or division of two integers, plus the addition or subtraction of an integer from the result. Hard sentences contained four components: the multiplication or division of two integers, plus the addition or subtraction of the result of a second multiplication or division of two integers, followed by the addition or subtraction of an integer from the result. Examples of an easy and hard math sentence are "Is $(8 \times 2)+4=20$ ?" and "Is $(2 \times 3)+(4 / 1)-5=5$ ?" respectively. The numbers used in both components were integers between 1 and 10. Numbers, rather than words, were used for the sentence verification task because Campbell's (1994) work had shown a decline in performance when words were used for a math task.

There were 108 math sentences developed for the sentence verification task. The sentences were used three times - once in each replication of the experimental conditions. As much as possible, within each of the 54 sentences used in each of the difficulty conditions, there were equal numbers of multiplication and division and of subtraction and addition facts, with half of the examples being correct and the other half incorrect. The incorrect answer was always within two units of the correct answer. The pairing of the items used in the math task with the storage items was random, and within each of the recall conditions, there was an equal number of correct and incorrect math sentences.

Three presentation format conditions were used in the present experiment, and difficulty of the sentence verification task was manipulated. The participants read out loud the math sentence as soon as it appeared and then verified its accuracy, while simultaneously remembering three or six items.

Two length of to-be-remembered item conditions (three and six items) combined with three presentation formats (picture, printed word, or spoken word) and two levels of sentence difficulty (easy or hard) resulted in 12 experimental conditions, which were replicated three times for a total of 36 trials. Within each replication, the order of the 12 experimental conditions was randomized. Items presented for storage were counterbalanced across length of to-beremembered items and sentence difficulty conditions, so that within each replication, different concepts were used as picture, printed word, and spoken word stimuli. Recall was scored as the proportion of items correctly remembered.

All the stimuli were displayed on a 15 -in. Apple flat screen monitor. Stimulus presentation and data collection were controlled by SuperLab running on a Power Mac G4 computer.
Procedure. All the participants were run individually in sessions of around $30 \mathrm{~min}$. They were given three practice trials in order to familiarize them with the task. They were asked to read out loud the math sentence as soon as it appeared and then to verify its accuracy, while simultaneously remembering a number of items (a total of three or six on each trial) presented in one of the three presentation format conditions. The processing and storage components of the task were interleaved. The math sentence appeared first and remained on the screen until the participant made a yes/no response, using the right or the left index finger. A single item from the storage task followed the participant's response. After $1 \mathrm{sec}$, a second math sentence appeared, followed by the second item from the storage task. A string of three question marks cued recall following presentation of the last storage item. The participants called out as many of the items as they could remember in any order, and their spoken responses were recorded by a research assistant while the computer recorded the RTs and error rates from the sentence verification task. When ready, the participant used a keypress to begin the next trial.

\section{Results}

Means were computed from the proportion of items correctly recalled and from correct RTs to the sentence verification for each participant across the three replications within each of the experimental conditions. Also recorded were the proportions of incorrect RT responses. Data for recall, RTs, and errors were treated separately with $3 \times 2 \times 2$ repeated measures analyses of variance (ANOVAs) to test for presentation format, difficulty of the processing task, and length of to-be-remembered items. The data from 3 participants were removed before analysis because, in some conditions, over half of their RT responses were longer than the $15.5-\mathrm{sec}$ cutoff for the trimmed means. The $F$ tests that are reported in all of the experiments discussed in this report include the GreenhouseGeisser correction, to protect against possible violation of the homogeneity assumption.

Figure 1 presents the means for item recall with 95\% confidence intervals. Consistent with previous findings, recall was strongly influenced by presentation format $\left[F(2,44)=131.62, p<.01, \eta^{2}=.86\right]$, number of to-beremembered items $\left[F(1,22)=649.52, p<.01, \eta^{2}=.97\right]$, and the format $\times$ number of items interaction $[F(2,44)=$ $\left.6.33, p<.01, \eta^{2}=.22\right]$. When only three items were to be remembered, spoken words were recalled almost perfectly (.95), with pictures somewhat lower (.87) and printed words quite low (.67). With six items to remember and more working memory capacity used, spoken words and pictures were nearly equal (.68 and .64, respectively), whereas printed words were the lowest (.49). Recall was lowest when items were presented as printed words and for long, rather than short, lists.

Varying the difficulty of the processing task also had an effect on recall $\left[F(1,22)=7.17, p<.02, \eta^{2}=.25\right]$, and the effect interacted with presentation format $[F(2,44)=$ $\left.9.21, p<.01, \eta^{2}=.29\right]$. Recall was higher when easy, rather than difficult, math sentences were processed, but only for picture and spoken word items. When printed words were recalled, processing difficulty had no effect.

The RT data presented in the middle panel of Figure 1 are trimmed means. Fewer than $5 \%$ of the responses 

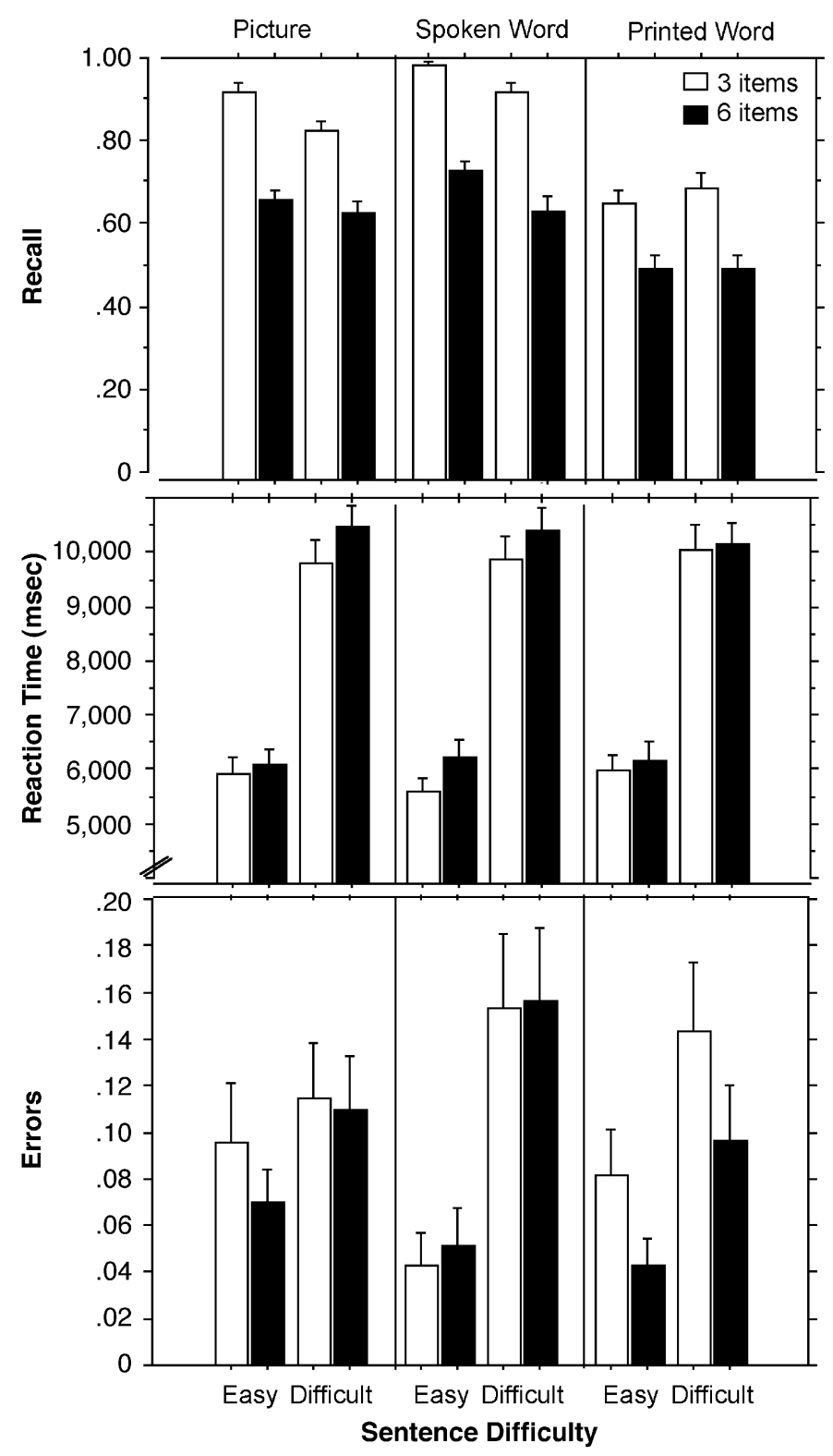

Figure 1. Mean scores for the format $\times$ sentence difficulty $\times$ list length interaction in Experiment 1. The error bars represent $95 \%$ confidence intervals. The top panels present the proportion of items recalled in the storage task, and the middle panels present the reaction times for the sentence verification task. The bottom panels present the proportion of errors made in the sentence verification task.

were in excess of $15.5 \mathrm{sec}$. No effect of presentation format on RT was obtained $(F<1)$. However, there were significant effects of sentence difficulty and number of items $\left[F(1,22)=263.56, p<.01, \eta^{2}=.92\right.$, and $F(1,22)=$ $8.70, p<.01, \eta^{2}=.28$, respectively]. The participants responded more rapidly when the sentence was easy $(5,990$ vs. $10,125 \mathrm{msec})$ and when the list length was short ( 7,862 vs. $8,253 \mathrm{msec})$. RTs were also affected by a small format $\times$ number of items interaction $[F(2,44)=$ $\left.4.15, p<.02, \eta^{2}=.16\right]$ and a sentence difficulty $\times$ for- mat $\times$ number of items interaction $[F(2,44)=4.89, p<$ $\left..01, \eta^{2}=.18\right]$. The interactions resulted from an absence of the short versus long list effect, with pictures in the easy processing condition and printed words in the difficult processing condition.

Error rates from the sentence verification task are shown in the bottom panel of Figure 1. Mean proportion of errors varied by sentence difficulty $[F(1,22)=12.50$, $\left.p<.01, \eta^{2}=.36\right]$ and by the interaction of format and sentence difficulty $\left[F(2,44)=7.72, p=.01, \eta^{2}=.26\right]$, 
but not by format $(F<1)$. More errors were made with hard than with easy sentences under all three formats, but the difference between hard and easy sentences was greatest with spoken words.

\section{Discussion}

Increasing the difficulty of the math task had a much larger effect on math task performance than on recall. The participants took almost twice as long to respond to difficult, rather than easy, math sentences and made twice as many errors. Increasing the difficulty of the math sentence did, however, also produce a small effect on recall, but for only two of the three presentation formats: pictures and spoken words.

The fact that processing task difficulty had much less of an effect on printed words, in comparison with the other presentation formats, may suggest that printed words use fewer resources, perhaps because they are not attended to as closely. The reduction in recall of pictures and spoken words with an increase in the difficulty of the processing task may be an indication that those formats are, in fact, using more rather then less, processing resources than are printed words. As the use of resources is increased with a more difficult math task, the resources available for pictures and spoken words decline, and performance suffers. An increase in resource use with a difficult math task might have little effect on printed words if they use fewer resources. This explanation is also consistent with the obtained interaction between format and list length. Although increasing the number of to-beremembered items from three to six lowered recall of printed words (from .67 to .49), it had an even greater effect on spoken words (from .94 to .68) and on pictures (from .87 to .64). Together, these findings suggest that printed words receive less attention and are, thus, not disrupted as much by an increase in the demand for resources from some other task. We tested this possibility more directly in Experiments 4 and 5.

Were processing and storage totally independent, as is assumed by the functional representation hypothesis (Oberauer, 2002), no effect of processing difficulty on the recall of stored items would be expected. The effect was, however, small and may suggest that the pool for resources shared among different functional representation levels is also small.

Format and span effects were consistent with the results in our previous studies. There was a lower recall of printed words than of pictures and spoken words and, in some conditions, an advantage of spoken words over pictures. When more items were held in memory, there was a lower recall and a longer RT, but number of to-be-remembered items had no influence on processing accuracy.

\section{EXPERIMENT 2}

In the second experiment, we changed the processing task from an arithmetic task to one involving visual/spatial reasoning. In all prior work, it was possible that the par- ticipants, although engaging the central executive in the actual execution of each item in the processing task, also used the verbal buffer to store intermediate results. Such a system would cause interference with the storage of words, but not with the storage of pictures, because presumably, they are stored in an alternative visual buffer. Recall of spoken words may not have shown as much interference from the processing task as printed words did because of the modality effect. If the nature of the processing task underlay the effect of presentation format, a change to a visually based processing task should alter the obtained effects of presentation format. Such a change might be expected to improve performance with words and to interfere with recall of pictures. This hypothesis was tested in the present experiment.

The visual/spatial-processing task was a sentence verification task that required the participants to make an inference from background items for which visual objects were used. Figure 2 presents examples of the three kinds of problem-solving items that were used-probability judgments with colors and with shapes and spatial relations between objects. These materials were used to develop a sentence verification task involving yes/no RTs. As in Experiment 1, the sentences were questions, and the difficulty of the task was varied by manipulating the number of lines of background material that needed to be considered before an inference was made. Two lines of material were used for the easy task, whereas the difficult task had four. These items were a modification of the problemsolving items originally developed by Goolkasian (1996).

The data collected in this experiment were also analyzed to examine the long-term modality effect. By comparing the recall of the final item presented on each trial across presentation format, we would expect to obtain an advantage of auditory (spoken word) over visual presentation (printed word; Gardiner et al., 1983; Greene, 1985; Greene \& Crowder, 1986; Greene et al., 1988; Penney, 1989). Modality effects have been assumed to be the result of an advantage for auditory input in working memory and to depend on the semantic properties of the items to be remembered and the distractor items. The effect is obtained when these items are semantically different (Greene, 1985), as is the case in our experiments. Our analysis is an attempt to determine whether or not the advantages we have found for spoken words over printed words are due, in part, to this modality effect. That is, the modality effect may account for some of the obtained advantage of spoken words over printed words by producing a higher level of recall for the last item presented when a longer sequence of items (six) is to be remembered. Such a modality effect would be evident if spoken words (auditory presentation) were superior to printed words and pictures (visual presentations) for that sixth item.

In sum, the present experiment is an attempt to determine whether the advantage of spoken words and of pictures over printed words is due to two different mechanisms. Long-term modality effects may underlie the advantage 
Easy Sentences

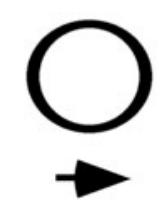

Is the circle below the arrow?

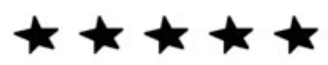

C

Are circles less likely?

"red"

"blue"

Are red squares more likely?
Difficult Sentences

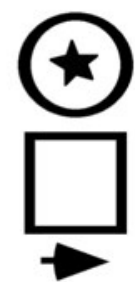

Is the square below the star?

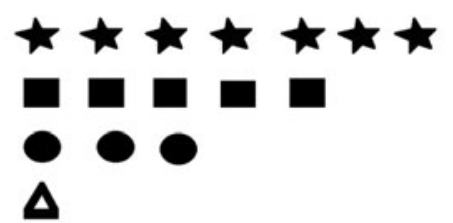

Are stars most likely?

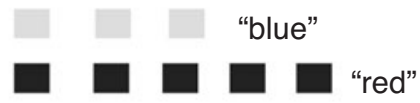

"yellow"

$$
\text { "green" }
$$

Are red squares least likely?

Figure 2. Examples of the problem-solving items used in the sentence verification task in Experiment 2. The top panel presents an item from the spatial relations between objects, and the other two panels have items from probability judgments with shapes and colors. For the bottom panels, the colors of the squares are printed in quotes.

for spoken words, whereas the specific processing task used in our experiments may underlie the advantage for pictures.

In all other respects, the experiment was the same as Experiment 1. The storage task required the participants to remember three or six items presented as pictures, spoken words, or printed words.

\section{Method}

Participants. The participants were 28 men and women students drawn from the same population as that in the previous experiment. Participation was, however, restricted to those with normal or corrected-to-normal color vision.

Materials. The processing task asked the participants to verify the accuracy of a sentence by referring to two or four lines of objects. Fifty-four stimuli were developed for each of the difficulty conditions. As much as possible within each of the difficulty conditions, there were equal numbers of items from the three kinds of problem-solving items - probability judgments with color and shape and spatial relations. For probability judgments with color, red, blue, yellow, and green squares were used that varied in number from one to seven, and the participant was required to make a judgment about whether a certain color was more or less likely. For probability judgments with shapes, stars, squares, circles, and triangles were used that varied in number from one to seven, and the participants were required to make a judgment about whether a shape was more or less likely. Spatial relation items presented two or four visual objects (star, arrow, square, and circle), and the participants were required to judge whether one object was below, on top of, or in another object. Equal numbers of the sentences were correct and incorrect. Each of the 108 sentences was used three times - once within each of the replications of the recall conditions. In all other respects, the stimulus materials were the same as those in the previous experiment. The same items were used in the storage task, and all materials were controlled by SuperLab.

Procedure. The procedure was the same as that in Experiment 1. The participants read out loud the sentence as soon as it appeared and then verified its accuracy while simultaneously remembering up to three or six items. Length of to-be-remembered items (three or six) was combined with three presentation formats (picture, spoken word, or printed word) and two levels of sentence difficulty (easy or hard). Each of the 12 experimental conditions was replicated three times, for a total of 36 recall trials.

\section{Results}

The data from the 28 participants were analyzed with a $3 \times 2 \times 2$ repeated measures ANOVA. Figure 3 presents the means from the data collected. In accord with previous findings, recall was strongly influenced by presentation format $\left[F(2,54)=75.37, p<.01, \eta^{2}=.74\right]$ and by length of to-be-remembered lists $[F(1,27)=320.67$, $\left.p<.01, \eta^{2}=.92\right]$. The format $\times$ length interaction was 

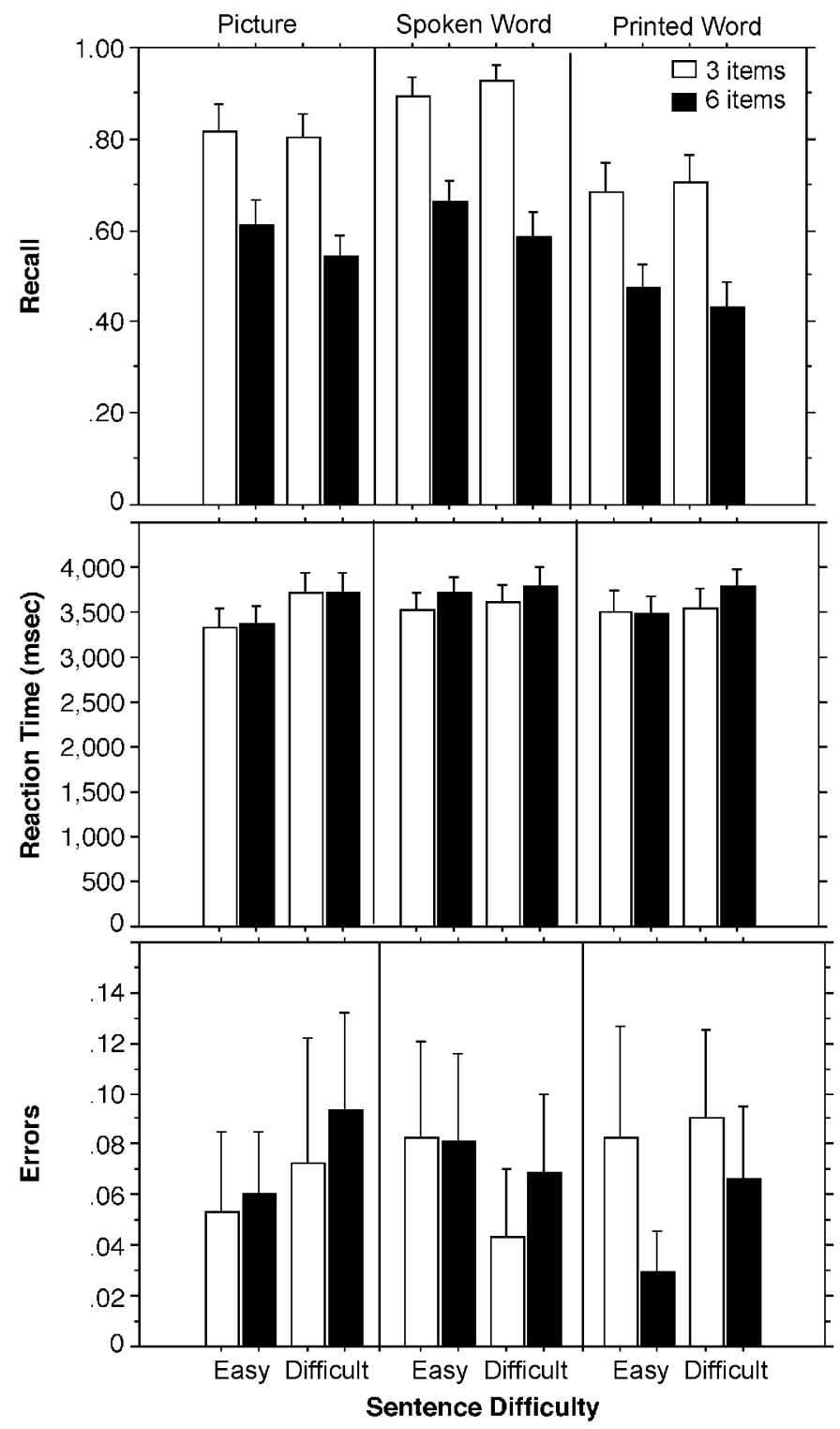

Figure 3. Mean scores for the format $x$ sentence difficulty $\times$ list length interaction in Experiment 2. The error bars represent $95 \%$ confidence intervals. The top panels present the proportion of items recalled in the storage task, and the middle panels present the reaction times for the sentence verification task. The bottom panels present the proportion of errors made in the sentence verification task.

not significant $[F(2,54)=2.71, p=.08]$. Recall was lowest when the items were presented as printed words, and long lists were not recalled as well as short lists.

Varying the difficulty of the processing task also had an effect on recall $\left[F(1,27)=4.31, p<.05, \eta^{2}=.14\right]$, and the effect interacted with list length $[F(2,54)=18.79$, $\left.p<.01, \eta^{2}=.41\right]$. With short lists, task difficulty made no difference (the mean proportion correct was .80 and .81 for easy and hard tasks, respectively), but with long lists, the easier task resulted in better recall than the hard task did (.58 and .52 , respectively).
The RT data presented in the middle panel of Figure 3 are trimmed means. Fewer than $5 \%$ of the responses were in excess of $6 \mathrm{sec}$. In accord with the previous experiment, task difficulty had a strong effect on RTs $[F(1,22)=$ $38.27, p<.01, \eta^{2}=.59$ ], and it interacted significantly with format $\left[F(2,54)=13.77, p<.01, \eta^{2}=.34\right]$ and with format and list length $[F(2,54)=4.79, p<.02$, $\left.\eta^{2}=.15\right]$. The participants responded more quickly to the easy than to the difficult processing task. Also, with the hard task, RTs were equivalent across the format conditions. With the easy task, RTs were shorter when pictures 
were presented. The analysis also showed significant main effects of format and length of lists $[F(1,54)=$ $7.99, p<.01, \eta^{2}=.23$, and $F(1,27)=12.45, p<.01$, $\eta^{2}=.32$, respectively]. The participants responded more slowly when spoken words were presented and quickest when pictures were presented and the number of storage items was small.

Error rates from the sentence verification task are shown in the bottom panel of Figure 3. Error rates represented about $7 \%$ of the responses across the three presentation formats. There were no significant variations as a function of presentation format, length of lists, or task difficulty $(F \mathrm{~s}<1)$. However, mean proportion of errors was influenced by the interaction between format and length of list $[F(2,54)=3.82, p<.05]$. With pictures and spoken words, error rates were equivalent for short and long lists, but with printed words, more errors were made with short (.10) than with long (.05) lists. There was also a significant interaction between task difficulty and format $[F(2,54)=6.68, p<.01]$. For pictures and printed words, more errors were made with the hard task, whereas with spoken words, more errors were made with the easy task.

To examine long-term modality effects, we looked at average recall of the last item that appeared in each of the experimental conditions. These means are shown in Table 1 . These data were analyzed with a $3 \times 2 \times 2$ repeated measures ANOVA. There was a significant interaction between format and number of items $[F(2,54)=$ $3.77, p<.03]$. When only three items were to be remembered, recall of last pictures (.98), spoken words (.97), and printed words (.98) were the same. When six items were to be remembered, both pictures (.96) and spoken words (.98) resulted in better performance than did printed words (.92). Planned comparisons were conducted to test for significance and showed no difference between pictures and spoken words $[F(1,54)=1.43, p=$ .24]. Pictures and spoken words were recalled better than printed words $[F(1,54)=7.60, p<.01]$.

None of the other effects were significant. There were no main effects of format, number of items to be remembered, or task difficulty $[F(2,54)=1.74, p=.18$, $F(1,27)=3.79, p=.06$, and $F<1$, respectively].

\section{Discussion}

In accord with the first experiment, manipulating task difficulty had a larger effect on performance in the RT

Table 1

Proportion of Times Across Conditions in Experiment 2 That the Last Item in the List Was Recalled

\begin{tabular}{lcccc}
\hline \multirow{2}{*}{ Task } & List & Picture & Spoken Word & Printed Word \\
\cline { 3 - 5 } Easy & Short & 1.00 & .95 & 1.00 \\
& Long & .95 & .98 & .94 \\
\multirow{2}{*}{ Difficult } & Short & .96 & .99 & .98 \\
& Long & .96 & .99 & .91 \\
\hline
\end{tabular}

task than in the recall task. Changing the processing task, from math sentences to a reasoning task based on visual judgments, did not alter the major findings with respect to task difficulty. Similarly, changing the type of processing task did not alter the presentation format effect. Pictures and spoken words were still recalled at a higher level than were printed words. The only difference between the findings in Experiments 1 and 2 was the absence of a presentation format $\times$ task difficulty interaction in the recall data. In Experiment 2, the hard processing task was associated with lower recall in all of the format conditions.

The reanalysis of the data showed that recall of the last item was strong for all of the conditions in the experiment. In accord with previous findings of the long-term modality effect, recall of spoken words was better than that of printed words, but the advantage was slight (.06). This small difference may have contributed to the presentation format effect observed in prior experiments but, by itself, is too small to explain the previous findings. Furthermore, a similar advantage was obtained when recall of pictures was compared with recall of printed words, and pictures and spoken words did not differ.

The present findings rule out the type of processing task and the traditional modality effect as explanations for the superior performance of pictures and spoken words over printed words. Our visual-processing task did not alter the relative performance with the three formats, and auditory presentation did not produce superior performance over both forms of visual presentation for the last item in long sequences. It is, of course, still possible that the mechanism producing superior performance in working memory is not the same for pictures and spoken words, but at present, we think it more parsimonious to assume that a common mechanism underlies the superiority of both pictures and spoken words over printed words.

The present results again support the notion that there is some sharing of resources between storage and processing tasks in working memory. With six items to remember, recall was lower when the more difficult processing task was presented. In addition, RTs to the processing task were influenced by modality/format. Errors were also influenced by the number of items held in memory. Storage and processing are not independent. However, task difficulty had a relatively small effect on recall, and similarly, the effect of presentation format on RTs in the processing task was quite small, relative to the effect of task difficulty and list length.

\section{EXPERIMENT 3}

With the exception of an interaction of format and task difficulty with the recall data in Experiment 1, the effects of presentation format remain consistent regardless of the difficulty or type of processing task. Something more than the type of processing task and the long-term modality effect produces the better recall of items presented as pictures and spoken words, rather than as printed 
words. We propose that there is a common mechanism that underlies this better performance, rather than a modalityspecific explanation for spoken words and some other explanation for pictures. Our next two experiments tested an attention allocation hypothesis that focuses on the lower performance with printed words, rather than on the superior performance with pictures and spoken words.

Printed words are hypothesized to be at some disadvantage in recall tasks such as ours because, relative to other presentation formats, they ordinarily demand less conscious attention to process. Printed words have been known to be processed with very little conscious attention in some situations. Examples of this are in investigations of the Stroop effect, although one would expect similar results for spoken words in the present experiments if automatic processing was responsible for the findings (Besner, Stolz, \& Boutilier, 1997; Mayer \& Kosson, 2004; Stroop, 1935). Our hypothesis is simply that printed words are not attended to as closely as are spoken words and pictures and that this difference in attention accounts for the advantage obtained for spoken words and pictures over printed words. This hypothesis is consistent with other work suggesting that an alerting effect found for auditory stimuli is dependent on the central allocation of attention and occurs for some visual, as well as auditory, stimuli (Turatto et al., 2002). The lower performance with printed words may be due to a lower level of conscious attention and less effortful processing (Hasher \& Zacks, 1979), an absence of an alerting effect, for printed words. It is not that attention is not paid to printed words, for clearly they are recalled well. The attention allocation hypothesis proposes that in this task, printed words require less attention for processing, relative to the other formats, and as a result are not recalled as well.

To test the attention allocation hypothesis, we made attention to all the items presented more equivalent by requiring the participants to articulate each item in the storage task. Saying items aloud should increase the allocation of attention to printed words and improve performance. The advantage of spoken words and pictures should be reduced under the present conditions, in which all the items must receive enough attention to result in accurate articulation.

\section{Method}

The participants were 27 men and women volunteers drawn from the same participant pool as that in the previous experiments. The same materials as those in Experiment 2 were used again.

All the participants were run individually in sessions of around $30 \mathrm{~min}$. They were given three practice trials to familiarize themselves with the task. In addition to articulating the sentence presented in the processing task, the participants were also asked to say out loud each presented picture, spoken word, or printed word as it was presented. In all other ways, the procedure was the same as that in Experiment 2.

\section{Results}

Data from the 27 participants were analyzed with a $3 \times 2 \times 2$ repeated measures ANOVA. Figure 4 presents the means from the data collected. Recall was significantly influenced by format $\left[F(2,52)=6.12, p<.01, \eta^{2}=.19\right]$, list length $\left[F(1,26)=316.30, p<.01, \eta^{2}=.92\right]$, and the interaction of format and list length $[F(2,52)=6.20, p<$ $\left..01, \eta^{2}=.19\right]$. Pictures (.75) and spoken words (.75) were again recalled better than printed words (.70), although the gap between these different conditions was greatly diminished, as compared with the results in Experiments 1 and 2 and in prior work (Goolkasian \& Foos, 2002, 2005). Moreover, a test of simple effects shows that the presentation format effect was present only for long lists $(p<$ $.01)$. Effects of presentation format were not obtained when the participants recalled short lists $(p<.16)$.

In accord with the results of Experiment 2, task difficulty had a significant effect on the proportion of items recalled $\left[F(1,26)=13.71, p<.01, \eta^{2}=.34\right]$, and the effect interacted with item length $[F(1,26)=9.31, p<.01$, $\left.\eta^{2}=.26\right]$. When only three items were to be remembered, recall was the same (.86) with both easy and hard processing tasks, but with six items, easy processing (.65) resulted in higher recall than harder processing did (.57). Presentation format was not found to interact with task difficulty $(F<1)$, nor was there a presentation format $\times$ list length $\times$ task difficulty interaction $[F(2,52)=2.33$, $p<.11]$.

The RT data, presented in the middle panel of Figure 4, are trimmed means. Fewer than $2 \%$ of the responses were in excess of $7.5 \mathrm{sec}$. As was expected, the participants responded more quickly to the easy than to the difficult processing task $\left[F(1,26)=35.54, p<.01, \eta^{2}=\right.$ $.58]$. The analysis also showed a strong effect of presentation format $\left[F(2,52)=35.79, p<.01, \eta^{2}=.58\right]$ and a significant interaction between format and number of items $\left[F(2,52)=3.28, p<.05, \eta^{2}=.11\right]$. The significant effects of format resulted from the requirement to articulate the items presented in the recall task. RTs were delayed in the spoken word conditions, relative to the other presentation formats, because the participants needed to wait until the end of the presentation interval to hear the whole word so that they could articulate the item. As a result, RTs were delayed to the subsequent sentence in the processing task. In general, comparisons with Figure 3 show that articulation of the storage items caused a lengthening of RTs across all of the conditions.

Error rates from the sentence verification task are shown in the bottom panel of Figure 4. They represented about $7 \%$ of the responses across the three presentation formats. There were no significant variations as function of presentation format, length of lists, or task difficulty $[F(2,52)=1.46, p=.24$, and two $F \mathrm{~s}<1$, respectively $]$. However, mean proportion of errors was influenced by the interaction between format and length of list $[F(2,52)=$ $8.89, p<.05]$. With a three-item list, the fewest processing errors occurred with spoken words (.05, as compared with .08 and .09 for pictures and printed words, respectively), whereas with a six-item list, most errors occurred with spoken words (.09, as compared with .04 and .06 for pictures and printed words, respectively). There was also a significant interaction between task difficulty and format 

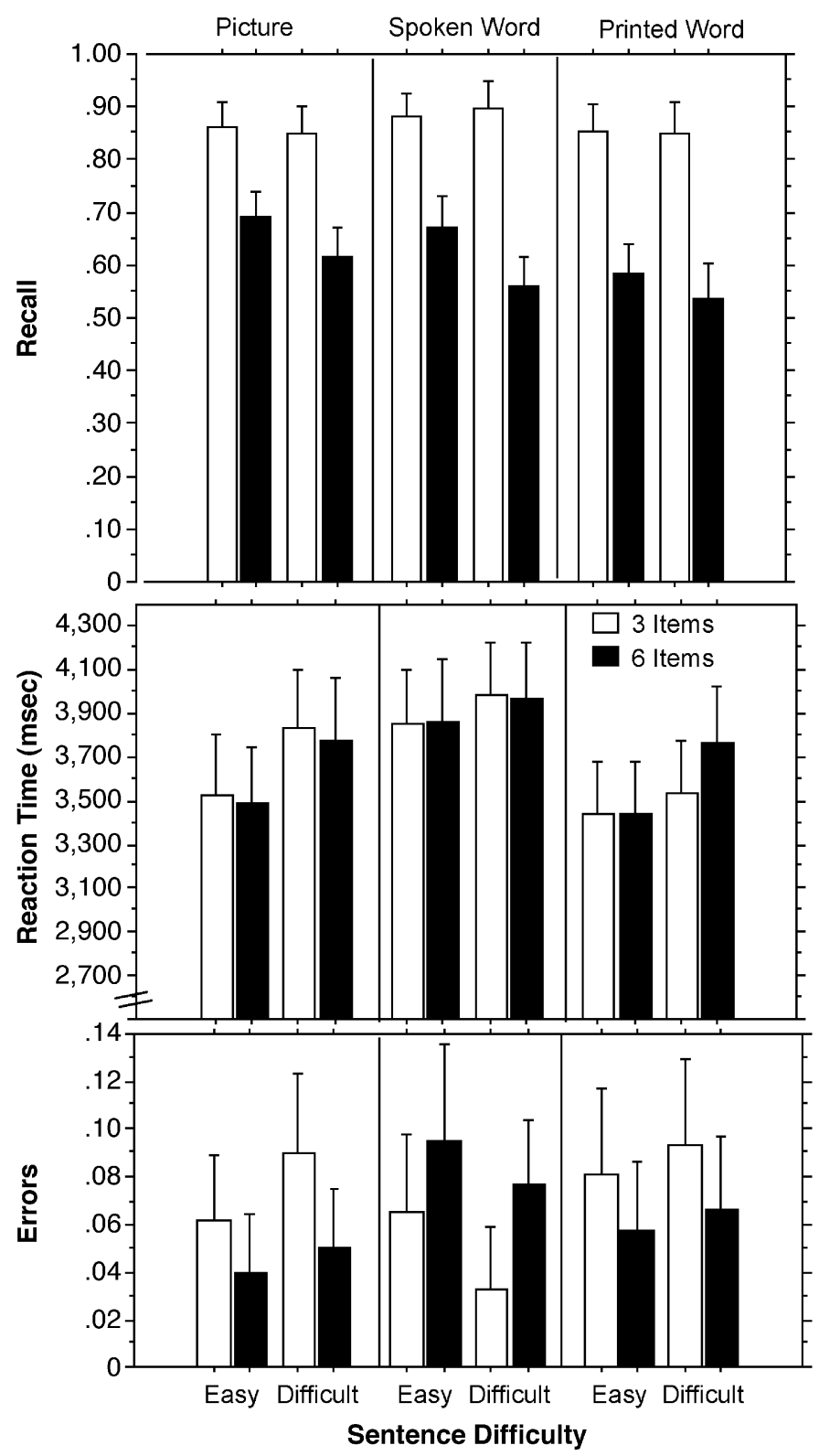

Figure 4. Mean scores for the format $\times$ sentence difficulty $\times$ list length interaction in Experiment 3. The error bars represent $95 \%$ confidence intervals. The top panels present the proportion of items recalled in the storage task, and the middle panels present the reaction times for the sentence verification task. The bottom panels present the proportion of errors made in the sentence verification task.

$[F(2,52)=4.02, p<.03]$. For pictures and printed words, more errors were made on the hard task, whereas for spoken words, more errors were made on the easy task.

\section{Discussion}

The present results lend support to the attention allocation hypothesis, although when long lists of items were used, printed words were still recalled at a lower level than pictures and spoken words. The average difference between mean performance with printed words and the other conditions in Experiment 1 was $.20(.78-.58)$ and, in Experiment 2, $.16(.73-.57)$, whereas in the present experiment, this difference was only $.05(.75-.70)$. Performance with printed words greatly increased (from .58 and .57 to .70 ) with the addition of articulation, whereas performance with pictures and spoken words did not.

It is, however, possible that articulation greatly increased the auditory processing of printed words and 
strengthened that representation. Of course, one might then expect to find that pictures would be processed in visual and auditory channels as they are articulated, whereas spoken words would still be processed in only one channel. Performance with pictures and spoken words, however, remained at the same high level as in previous studies. It does not appear to be the case that the number of channels involved in processing plays a major role in these continually obtained modality/format differences.

The longer RTs to the processing task when to-beremembered items were presented as spoken words were undoubtedly due to the addition of articulation. Articulating each presented item delayed responding to the processing task in the spoken word condition, because the participants had to wait to hear the whole word and often articulation could begin only at the end of the interval. This constraint was not present when the items were presented as pictures or printed words. It is probable that the unusual pattern of processing errors with spoken words (slightly more errors with the easy than with the hard processing task) might also be due to the need to wait before articulating the spoken word.

\section{EXPERIMENT 4}

To further test the attention allocation hypothesis, we increased attention by adding a mask to the presentation of the storage items and compared that degraded condition with normal presentation. Degradation should force participants to more closely attend to the items to discern their identity, although all the items could still be identified. Degraded stimuli have been shown to take longer to recognize and encode (Duncan \& Bourg, 1983; Perfetti, 1985; Perfetti \& Roth, 1981; Sternberg, 1967) and would normally be expected to lower performance. The attention allocation hypothesis, however, predicts no such lowering for printed words that are degraded, since this demands closer attention and printed words ordinarily receive less attention. Under some conditions, memory for degraded printed words could be better than memory for normally printed words, since the former requires more attention. For pictures and spoken words, however, degrading should reduce performance.

\section{Method}

Participants. The participants were 27 men and women students drawn from the same population as that in the previous experiments. The participants were restricted to those with normal or corrected-to-normal color vision.

Materials. With the exception of 3 items, the 54 items used in the recall task were the same as the ones used in the previous experiments. For this experiment, angel, shovel, and baby were used, instead of cheese, pie, and blender. The items were obscured by superimposing a $5 \times 5 \mathrm{~cm}$ visual mask (a series of diagonal lines) on the picture and printed word stimuli and mixing an auditory mask ( $440-\mathrm{Hz}$ tone) into the spoken word sound file. The spoken word sound file and the auditory mask were integrated via the mix function of SoundEdit Pro (Version 2) software. Figure 5 presents examples of the visual stimuli.

Only the 54 stimuli developed for the easy processing condition in Experiment 2 were used. Those stimuli included equal numbers of

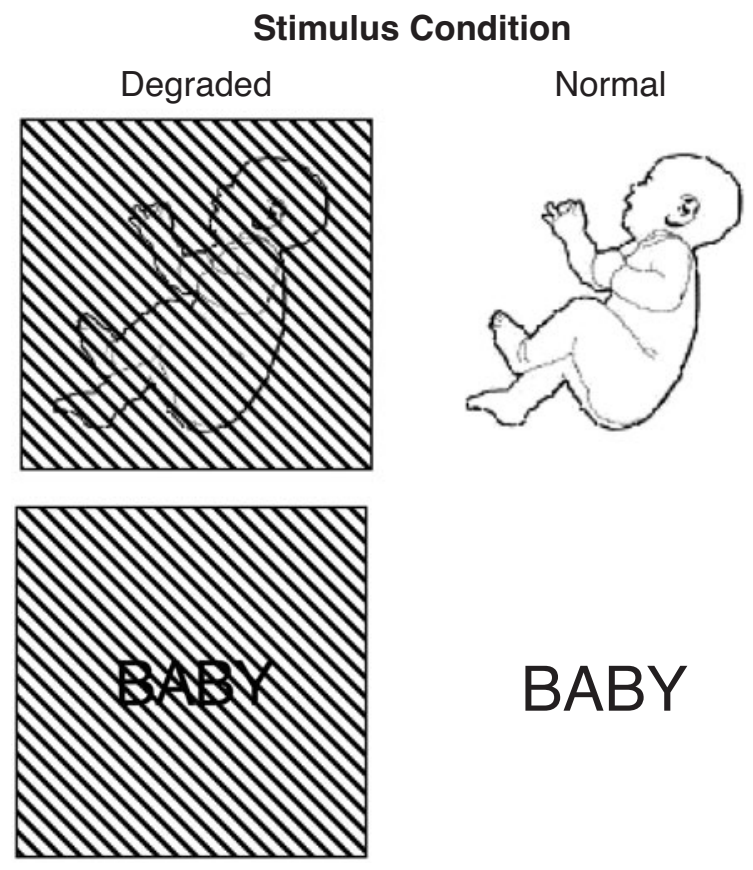

Figure 5. Examples of the stimulus items from the degraded and normal conditions used in the storage task in Experiment 4.

items from the three kinds of problem-solving items - probability judgments with color and shape and spatial relations.

Procedure. The procedure was the same as that in Experiment 2. The participants read out loud the sentence as soon as it appeared and then verified its accuracy while simultaneously remembering up to three or six items. The items in the storage task were either degraded or nondegraded (the item appeared without the mask). Presentation of the degraded and nondegraded items was blocked within each of the three replications, and within each block there was a random arrangement of presentation format (picture, spoken word, or printed word) and list length (three or six). All the participants were run individually in sessions of around $30 \mathrm{~min}$, and they were given five practice trials to familiarize themselves with the task. In all other ways, the procedure was the same as that in Experiment 2.

\section{Results}

The data from the 27 participants were analyzed with a $3 \times 2 \times 2$ repeated measures ANOVA. Figure 6 presents the means from the data collected. As in the prior experiments, recall was influenced by presentation format and number of items $\left[F(2,52)=40.96, p<.01, \eta^{2}=.61\right.$, and $F(1,26)=349.53, p<.01, \eta^{2}=.93$, respectively]. Pictures (.68) and spoken words (.71) resulted in better recall than printed words did (.56), and a higher proportion of items was recalled when only three items were presented. Significantly more items were recalled under the normal presentation condition (.68) than under the degraded condition $\left[.62 ; F(1,26)=24.07, p<.01, \eta^{2}=.48\right]$.

However, the negative effect on recall of degrading the stimuli varied as a function of presentation format $\left[F(2,52)=11.38, p<.01, \eta^{2}=.31\right]$, of number of items $\left[F(1,26)=5.02, p<.03, \eta^{2}=.16\right]$, and of presentation format and number of items $[F(2,52)=3.26, p<.05$, 

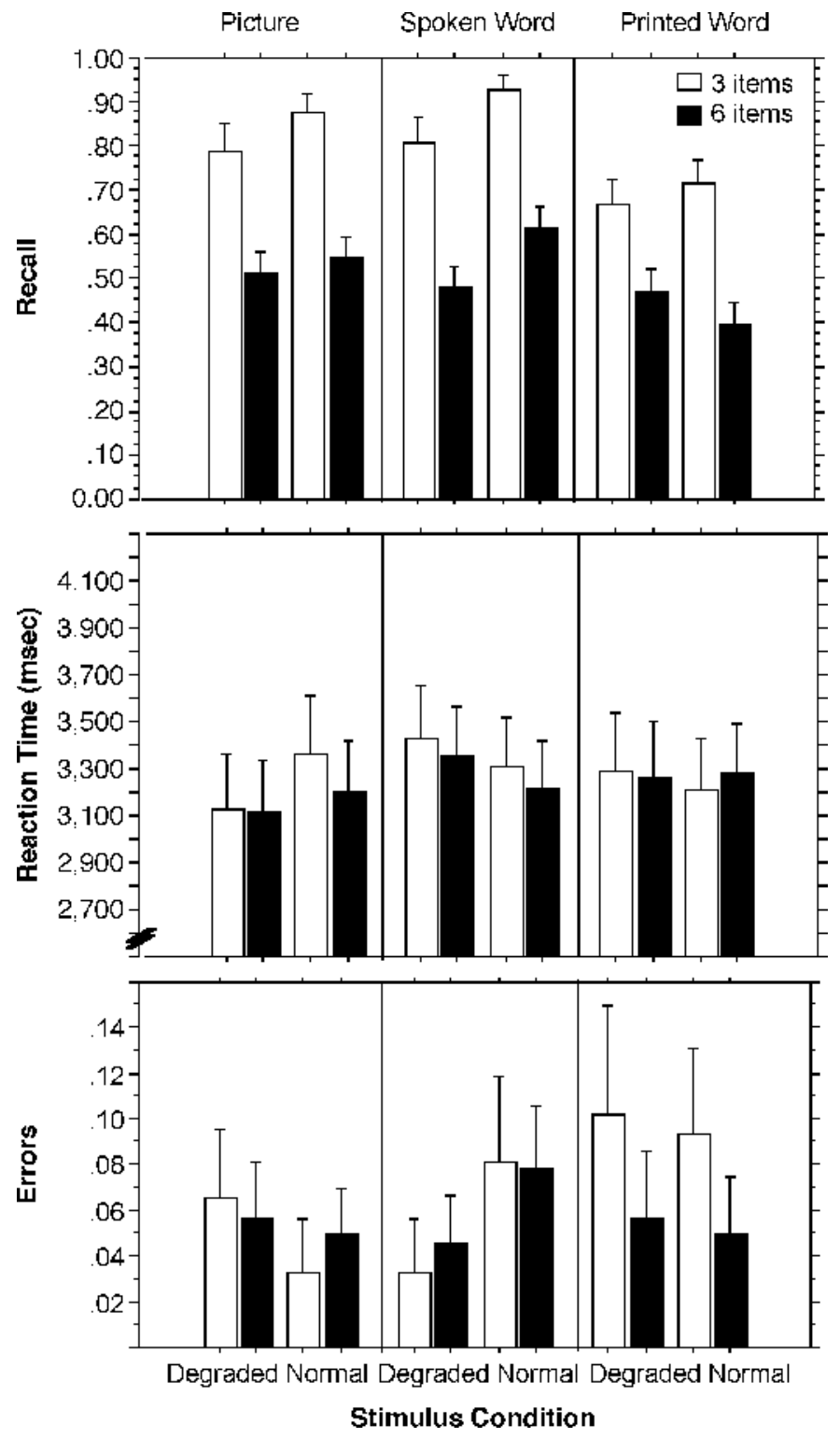

Figure 6. Mean scores for the format $\times$ stimulus condition $\times$ list length interaction in Experiment 4. The error bars represent $95 \%$ confidence intervals. The top panels present the proportion of items recalled in the storage task, and the middle panels present the reaction times for the sentence verification task. The bottom panels present the proportion of errors made in the sentence verification task.

$\left.\eta^{2}=.11\right]$. Degrading the stimuli lowered performance with pictures and spoken words, but not necessarily with printed words - it depended on the length of the list. Degrading the stimulus items lowered performance to a greater extent when only three items were to be remembered. When six items were to be remembered, degrading lowered performance with pictures and spoken words but actually increased performance for printed words (from .39 to .47). A planned comparison within the interaction confirmed the significance of the increase in recall for printed words in the degraded condition $(p<.01)$. Also noteworthy is the fact that recall of the degraded stimuli when six items were to be remembered was the same across presentation formats: Mean recall for pictures was .51 and .47 for both spoken and printed words. Planned comparisons showed that there were no differences between recall of pictures and printed words $(p=$ .14 ) and spoken words and printed words ( $p=.26$ ).

The RT data presented in the middle panel of Figure 6 are trimmed means. Fewer than $2 \%$ of the responses were 
in excess of $6.5 \mathrm{sec}$. Presentation format and the interaction between format and stimulus condition significantly influenced RTs $\left[F(2,52)=6.18, p<.01, \eta^{2}=.19\right.$, and $F(2,52)=5.73, p<.01, \eta^{2}=.18$, respectively]. RTs were slightly shorter for pictures, particularly in the degraded condition. This finding of shorter RTs for pictures was also found in Experiment 2 for the easy processing task.

Error rates from the sentence verification task are shown in the bottom panel of Figure 6. Error rates represented about $7 \%$ of the responses across the three presentation formats. Error rates were influenced by format and by the interaction between format and number of items $[F(2,52)=3.69, p<.04$, and $F(2,52)=5.44, p<$ .01 , respectively]. More processing errors were made when printed words were presented, particularly when only three items were presented. The error rate was not influenced by the presentation of degraded stimuli $(F<1)$, nor was the stimulus condition found to interact with any of the other variables.

\section{Discussion}

The present results strongly support the attention allocation hypothesis. Degraded pictures and spoken words were recalled at lower levels than normal pictures and spoken words were. For printed words, however, degrading made no difference when only three items were presented and actually improved performance when six items were to be remembered. The attenuation of attention that normally occurs with printed words could not occur to as great an extent when those words were more difficult to perceive. Degradation required the participants to allocate additional attention to these items. As a result, the effects of presentation format were greatly diminished.

As in Experiments 1, 2, and 3, the present results also support the hypothesis that resources are shared between processing and storage tasks, although that sharing may be minimal. Both RTs and errors on the processing task were influenced by modality/format, but not by the stimulus condition.

It is also possible that the effects of presentation format on the processing task may have resulted from the participants' attempts to rehearse the items presented in the recall task. Rehearsal would be more likely in the word conditions when a few items were held in storage. Findings of elevated error rates with words and reduced RTs with pictures support such an interpretation.

\section{GENERAL DISCUSSION}

The present work addresses the question of why some types of presentation modalities/formats result in better recall from working memory than other types do (Goolkasian $\&$ Foos, 2002, 2005). We examined the advantages of pictures and spoken words over printed words by varying the type and difficulty of the processing task and by manipulating the allocation of attention to presented items in the storage task. The present results also support models of working memory in which resources are shared between processing and storage tasks.
In terms of shared resources, changes in the difficulty of the processing task resulted in a decrease in recall for pictures and spoken words, but not for printed words, only in Experiment 1. In Experiment 2, recall of six items was lower when the more difficult processing task was presented. In addition, RTs to the processing task were influenced by modality/format. Finally, errors made on the processing task were influenced by the number of items stored in memory. These findings show that storage and processing are not completely independent, as some theorists have suggested (Oberauer, 2002), although the small effect sizes, relative to other effects in the analyses, suggest that the resources shared may be far less than the resources devoted solely to storage and processing. These findings partially support the central executive model of working memory proposed by Baddeley and colleagues (Baddeley \& Hitch, 1974; Baddeley \& Logie, 1999).

For presentation format effects, changing the processing task from math sentences to a reasoning task based on visual judgments did not alter the modality/format effect. Pictures and spoken words were still recalled at a higher level than were printed words. This finding argues against any explanation that may claim that the nature of the processing task underlay the effect of presentation modality/format. Had this been the case, a change to a visually based processing task should have altered the obtained effects of presentation format and should have improved performance with words and interfered with recall of pictures. These effects did not occur. We did, however, find that RTs were influenced in a small but consistent way by presentation format. When visual judgments were used as the basis for the processing task, RTs were slightly shorter when pictures were recalled, in comparison with all the other conditions. Exactly why this effect is occurring, however, is difficult to explain; however, it may result from participants' attempts to rehearse words to improve recall.

Is the advantage of spoken words due to the long-term modality effect (e.g., Greene, 1985)? In Experiment 2, we reanalyzed collected data to examine this possibility and found the same advantage for pictures and spoken words over printed words for the last item presented in a six-item list. This result suggests either that the modality effect is a contributor to the spoken word advantage and pictures have some other type of advantage or that the term modality effect is a misnomer. The advantage is not one of auditory presentation over visual presentation but is due to one common mechanism, the attenuation of attention for printed words.

Experiments 3 and 4 tested the allocation-of-attention hypothesis and showed that performance with printed words was greatly improved by articulation and, in some instances, by degrading presented stimuli. Articulation had no influence on the recall of pictures and spoken words, and degrading lowered performance with both of these stimulus types. The disadvantage for printed words (rather than the advantage for pictures and spoken words) lies in the allocation of attention. Printed words are not ordinarily given full attention. Attention to them is at- 
tenuated. Forcing attention to printed words improved performance.

The present results offer a new explanation for presentation format effects. The advantage of auditory presentation over visual and the advantage of pictures over printed words may, in part, be due to the disadvantage for printed words. There is a tendency, with stimulus items such as the ones used in these experiments, to extend more effort and attention toward the processing of materials presented as pictures and spoken words, rather than as printed words (e.g., Melara \& O’Brien, 1987; Patching \& Quinlan, 2002; Posner et al., 1976; Turatto et al., 2002). With printed words, attention is attenuated. When individuals do focus attention on printed words, performance improves, and printed words are then remembered as well as items presented in other modes and formats.

\section{REFERENCES}

BAdDEley, A. D., \& Hitch, G. J. (1974). Working memory. In G. H. Bower (Ed.), The psychology of learning and motivation: Advances in research and theory (Vol. 8, pp. 47-89). New York: Academic Press.

BADDELEY, A. D., \& LogIE, R. H. (1999). Working memory: The multiplecomponent model. In A. Miyake \& P. Shah (Eds.), Models of working memory (pp. 28-61). Cambridge: Cambridge University Press.

Barrouillet, P., Bernardin, S., \& CAMos, V. (2004). Time constraints and resource sharing in adults' working memory spans. Journal of Experimental Psychology: General, 133, 83-100.

Besner, D., Stolz, J. A., \& Boutilier, C. (1997). The Stroop effect and the myth of automaticity. Psychonomic Bulletin \& Review, 4, 221-225.

CAmpbell, J. I. D. (1994). Architectures for numerical cognition. Cognition, 53, 1-44.

Cowan, N., Saults, J. S., Elliott, E. M., \& Moreno, M. (2002). Deconfounding serial recall. Journal of Memory \& Language, 46, 153177.

Crowder, R. G. (1972). Visual and auditory memory. In J. F. Kavanagh \& I. G. Mattingly (Eds.), Language by ear and by eye: The relationships between speech and reading (pp. 251-275). Cambridge, MA: MIT Press.

Daneman, M., \& Carpenter, P. A. (1980). Individual differences in working memory and reading. Journal of Verbal Learning \& Verbal Behavior, 19, 450-466.

Duncan, E. M., \& Bourg, T. (1983). An examination of the effects of encoding and decision processes on the rate of mental rotation. Journal of Mental Imagery, 7, 33-55.

Gardiner, J. M., GARDiner, M. M., \& GregG, V. H. (1983). The auditory recency advantage in longer term free recall is not enhanced by recalling prerecency items first. Memory \& Cognition, 11, 616-620.

GoolKasian, P. (1996). Picture-word differences in a sentence verification task. Memory \& Cognition, 24, 584-594.

Goolkasian, P., \& Foos, P. W. (2002). Presentation format and its effect on working memory. Memory \& Cognition, 30, 1096-1105.

Goolkasian, P., \& Foos, P. W. (2005). Bimodal format effects in working memory. American Journal of Psychology, 118, 61-77.

Goolkasian, P., \& Park, D. C. (1980). Processing of visually presented clock times. Journal of Experimental Psychology: Human Perception \& Performance, 6, 707-717.

Greene, R. L. (1985). Constraints on the long-term modality effect. Journal of Memory \& Language, 24, 526-541.
Greene, R. L., \& Crowder, R. G. (1986). Recency effects in delayed recall of mouthed stimuli. Memory \& Cognition, 14, 355-360.

Greene, R. L., Elliott, C. L., \& Smith, M. D. (1988). When do interleaved suffixes improve recall? Journal of Memory \& Language, 27, 560-571.

Hasher, L., \& ZaCKs, R. T. (1979). Automatic and effortful processes in memory. Journal of Experimental Psychology: General, 108, 356388 .

Kroll, J. F., \& CoRrigan, A. (1981). Strategies in sentence-picture verification: The effect of an unexpected picture. Journal of Verbal Learning \& Verbal Behavior, 20, 515-531.

La Pointe, L. B., \& Engle, R. W. (1990). Simple and complex word spans as measures of working memory capacity. Journal of Experimental Psychology: Learning, Memory, \& Cognition, 16, 1118-1133.

MaYer, A. R., \& Kosson, D. S. (2004). The effects of auditory and visual linguistic distractors on target localization. Neuropsychology, 18, 248-257.

Melara, R. D., \& O'Brien, T. P. (1987). Interaction between synesthetically corresponding dimensions. Journal of Experimental Psychology: General, 116, 323-336.

OBerauer, K. (2002). Access to information in working memory: Exploring the focus of attention. Journal of Experimental Psychology: Learning, Memory, \& Cognition, 28, 411-421.

Oberauer, K., Demmrich, A., Mayr, U., \& Kliegl, R. (2001). Dissociating retention and access in working memory: An age-comparative study of mental arithmetic. Memory \& Cognition, 29, 18-33.

PaIvio, A. (1971). Imagery and verbal processes. New York: Holt, Rinehart \& Winston.

Paivio, A. (1975). Perceptual comparisons through the mind's eye. Memory \& Cognition, 3, 635-647.

Paivio, A. (1978). A dual coding approach to perception and cognition. In H. L. Pick \& E. Saltzman (Eds.), Modes of perceiving and processing information (pp. 39-51). Hillsdale, NJ: Erlbaum.

Patching, G. R., \& Quinlan, P. T. (2002). Garner and congruence effects in the speeded classification of bimodal signals. Journal of Experimental Psychology: Human Perception \& Performance, 28, 755-775.

Pellegrino, J. W., Rosinski, R. R., Chiesi, H. L., \& Siegel, A. (1977). Picture-word differences in decision latency: An analysis of single and dual memory models. Memory \& Cognition, 5, 383-396.

Penney, C. G. (1989). Modality effects and the structure of short-term verbal memory. Memory \& Cognition, 17, 398-422.

Perfetti, C. A. (1985). Reading ability. New York: Oxford University Press.

Perfetti, C. A., \& Roth, S. F. (1981). Some of the interactive processes in reading and their role in reading skill. In A. M. Lesgold \& C. A. Perfetti (Eds.), Interactive processes in reading (pp. 269-297). Hillsdale, NJ: Erlbaum.

Posner, M. I., Nissen, M. J., \& Klein, R. M. (1976). Visual dominance: An information-processing account of its origins and significance. Psychological Review, 83, 157-171.

SMith, M. C., \& MageE, L. E. (1980). Tracing the time course of pictureword processing. Journal of Experimental Psychology: General, 109, 373-392.

STERnBERG, S. (1967). Two operations in character recognition: Some evidence from reaction-time measurements. Perception \& Psychophysics, 2, 45-53.

STROOP, J. R. (1935). Studies of interference in serial verbal reactions. Journal of Experimental Psychology, 35, 643-662.

Turatto, M., Benso, F., Galfano, G., \& Umiltà, C. (2002). Nonspatial attentional shifts between audition and vision. Journal of Experimental Psychology: Human Perception \& Performance, 28, 628639. 


\begin{tabular}{ll} 
APPENDIX & $\begin{array}{c}\text { APst of Items } \\
\text { Ball }\end{array}$ \\
Bat & Blender \\
Bike & Cherries \\
Books & Umbrella \\
Boot & Football \\
Box & Guitar \\
Bus & Lighthouse \\
Car & Monkey \\
Cat & Mushrooms \\
Chair & Panda \\
Cheese & Pencil \\
Cow & Penguin \\
Crab & Pretzel \\
Dog & Rabbit \\
Whale & Radio \\
Egg & Toaster \\
Fish & Scissors \\
Fog & Snowman \\
Globe & Stapler \\
Horse & Toilet \\
House & Tractor \\
Key & Turtle \\
Plant & Calculator \\
Owl & Elephant \\
Pen & Balloons \\
Pie & Handcuffs \\
Pig & Microscope \\
revision accepted for publication July 21, 2004.) & Sunglasses \\
\hline & \\
\hline
\end{tabular}

